\title{
Research on Inspection Scheduling Optimization Model Based on Different Inspection Periods
}

\author{
Yan Linjing ${ }^{1}$ \\ Beijing Polytechnic \\ Beijing, 100176, China
}

\author{
Wang $\mathrm{Nan}^{2}$ \\ Beijing Polytechnic \\ Beijing, 100176, China
}

\begin{abstract}
Scheduling problem serves as a common problem both for production and daily life, and the scheduling system has been increasingly emphasized and widely applied with the help of information technology. In this paper, the inspection network of a chemical plant is abstracted as an undirected graph, and the shortest walk time between any inspection points is obtained by means of the Floyd algorithm based on graph theory. Moreover, based on the principle of humanized scheduling, the effective inspection work is defined, and a mathematical model is established and solved by combining the relative balance of the workload and minimizing the staff, and a better scheduling effect is realized.
\end{abstract}

Keywords-Floyd algorithm; Scheduling model; Inspection route; Optimization

\section{INTRODUCTION}

To improve the effectiveness of the allocation of human resources, it is necessary for a chemical plant to optimize the inspection scheduling under the premise that relatively balanced workload of the staff and the successfully completion of all inspection tasks, efficiency improvement and staff minimization. This problem is also prominent in other industries, which is the core issue of scheduling problems. Its connected graphs are as follows:

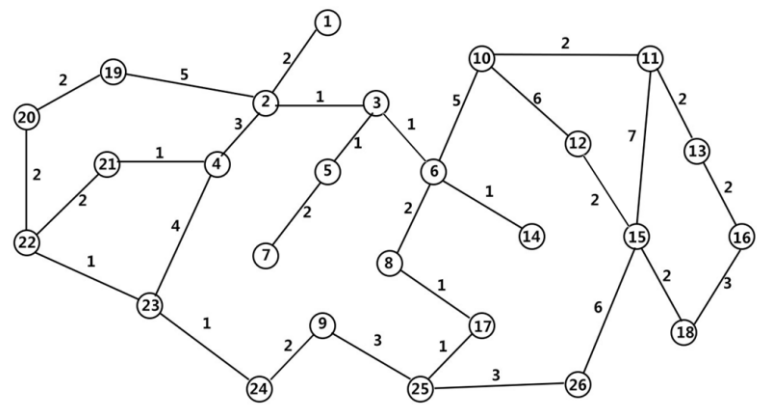

Fig. 1 Connected graph

We need to solve the following two problems: (1) According to the inspection network connectivity diagram of a chemical plant and the inspection cycle and inspection duration of each inspection point. According to the network connectivity diagram of a chemical plant and the inspection cycle of inspection points and the length of inspection, we adopt fixed work time, do not consider the rest time of the inspectors, use three shifts every day, work about 8 hours per shift, calculate the number of people needed in each class, and design the route of inspection and the timetable. (2) If the inspectors need to rest about 5 to 10 minutes for each inspection 2 hours, and they need to eat at about 12 noon and 6 p.m., each meal time is 30 minutes each time, still use three shifts every day, design a reasonable inspection line and inspection schedule, and calculate the number of staff required [2-3].

TABLE I BASIC DATA

\begin{tabular}{|c|c|c|}
\hline number & $\begin{array}{c}\text { Cycle } \\
\text { (minutes) }\end{array}$ & $\begin{array}{c}\text { Time of inspection } \\
\text { (minutes) }\end{array}$ \\
\hline XJ-0001 & 35 & 3 \\
\hline XJ-0002 & 50 & 2 \\
\hline XJ-0003 & 35 & 3 \\
\hline XJ-0004 & 35 & 2 \\
\hline XJ-0005 & 720 & 2 \\
\hline XJ-0006 & 35 & 3 \\
\hline XJ-0007 & 80 & 2 \\
\hline XJ-0008 & 35 & 3 \\
\hline XJ-0009 & 35 & 4 \\
\hline XJ-00010 & 120 & 2 \\
\hline $\mathrm{XJ}-00011$ & 35 & 3 \\
\hline $\mathrm{XJ}-00012$ & 35 & 2 \\
\hline $\mathrm{XJ}-00013$ & 80 & 5 \\
\hline XJ-00014 & 35 & 3 \\
\hline XJ-00015 & 35 & 2 \\
\hline $\mathrm{XJ}-00016$ & 35 & 3 \\
\hline XJ-00017 & 480 & 2 \\
\hline $\mathrm{XJ}-00018$ & 35 & 2 \\
\hline XJ-00019 & 35 & 2 \\
\hline $\mathrm{XJ}-00020$ & 35 & 3 \\
\hline XJ-00021 & 80 & 3 \\
\hline XJ-00022 & 35 & 2 \\
\hline XJ-00023 & 35 & 3 \\
\hline XJ-00024 & 35 & 2 \\
\hline XJ-00025 & 120 & 2 \\
\hline $\mathrm{XJ}-00026$ & 35 & 2 \\
\hline
\end{tabular}




\begin{tabular}{|c|c|c|}
\hline $\begin{array}{c}\text { Inspection } \\
\text { point A }\end{array}$ & $\begin{array}{l}\text { Inspection } \\
\text { point B }\end{array}$ & $\begin{array}{c}\text { Time consuming } \\
\text { (minutes) }\end{array}$ \\
\hline 1 & 2 & 2 \\
\hline 2 & 3 & 1 \\
\hline 2 & 4 & 3 \\
\hline 2 & 19 & 5 \\
\hline 3 & 5 & 1 \\
\hline 3 & 6 & 1 \\
\hline 4 & 21 & 1 \\
\hline 4 & 23 & 4 \\
\hline 5 & 7 & 2 \\
\hline 6 & 8 & 2 \\
\hline 6 & 14 & 1 \\
\hline 6 & 10 & 5 \\
\hline 8 & 17 & 1 \\
\hline 9 & 24 & 2 \\
\hline 9 & 25 & 3 \\
\hline 10 & 11 & 2 \\
\hline 10 & 12 & 6 \\
\hline 11 & 13 & 2 \\
\hline 11 & 15 & 7 \\
\hline 12 & 15 & 2 \\
\hline 13 & 16 & 2 \\
\hline 15 & 18 & 2 \\
\hline 15 & 26 & 6 \\
\hline 16 & 18 & 3 \\
\hline 17 & 25 & 1 \\
\hline 19 & 20 & 2 \\
\hline 20 & 22 & 2 \\
\hline 21 & 22 & 2 \\
\hline 22 & 23 & 1 \\
\hline 23 & 24 & 1 \\
\hline 25 & 26 & 3 \\
\hline
\end{tabular}

\section{ANALYSIS OF THE PROBLEM}

\section{A. Analysis of problem 1}

According to the analysis of the subject, the key to the problem is to find the right way to distribute the inspection route, which makes the human resources as small as possible and the effective workload is balanced. But because all the inspection cycles are different, in order to ensure that all points can complete the inspection normally, the minimum inspection cycle (35 minutes) can be considered as the inspection cycle of all points. However, many inspection points will be repeated and stopped without checking, resulting in low effective workload.

In order to design a people-centered rational inspection plan, we have defined the invalid workload and the effective workload.

Definition 1: invalid workload
The sum of residence time reserved for inspection points that do not require inspection during the inspection process.

Definition 2: effective workload

The total time of inspection and inspection during the inspection process.

\section{Definition 3: effective rate of work}

The ratio between effective workload and total workload.

According to the inspection cycle, F1 and F2 can be divided into two closed loop lines to find the best route respectively, and the inspection period is 35 minutes and 60 minutes respectively.

\section{B. Analysis of problem 2}

If the inspector takes 5-10 minutes off every 2 hours, the inspection cycle of the closed loop can be shortened. The inspection period can be changed to 35 minutes by 33 minutes, 60 minutes by 58 minutes. This ensures a 8 minute break every 2 hours.

If you consider dining at 12 noon and 6 p.m., the meal time is 30 minutes. You may consider that the inspection route of each person is not the only one. Take A for example, A 8 points to work, every 30 minutes a cycle, to 11 exactly the end of the integer period, at this time to 58 minutes for a cycle, and first test for 80 minutes, can be eaten for 30 minutes to eat, for $\mathrm{B}, 8$ to work, every 58 minutes a cycle, to 12 points is good integer cycle end, and I have had time to eat, then change to the original A shift, and rotate again at 6 p.m.

\section{MODEL ESTABLISHMENT AND SOLUTION}

We abstract the connection points of each inspection point into undirected weighted graphs in graph theory. Given the location coordinates of each inspection point and its adjacency relations, an undirected weighted graph $G(V, E)$ can be abstracted. $\mathrm{V}$ is the vertex set in the undirected weighting graph, representing all inspection points of a chemical plant; $\mathrm{E}$ is the edge set of the undirected weighted graph, and represents the route of any two adjacent inspection points. At the same time, the length of the route between the two adjacent inspection points is the weight of its corresponding edges. In order to process the undirected weighting graph by computer, we construct a weighted adjacency matrix $L$, and any element $L_{i j}$ of which represents the weight of the edge $\left(v_{i}, v j\right)$, that is, the distance between the adjacent inspection points $V_{i}$ and $V_{j}$. After the weighted adjacency matrix is obtained, the shortest path length matrix between any two cruising points $D$ is obtained according to the Floyd algorithm, and the element $t_{i j}$ is the shortest path length between arbitrary two points vi and vj [5-6]. 


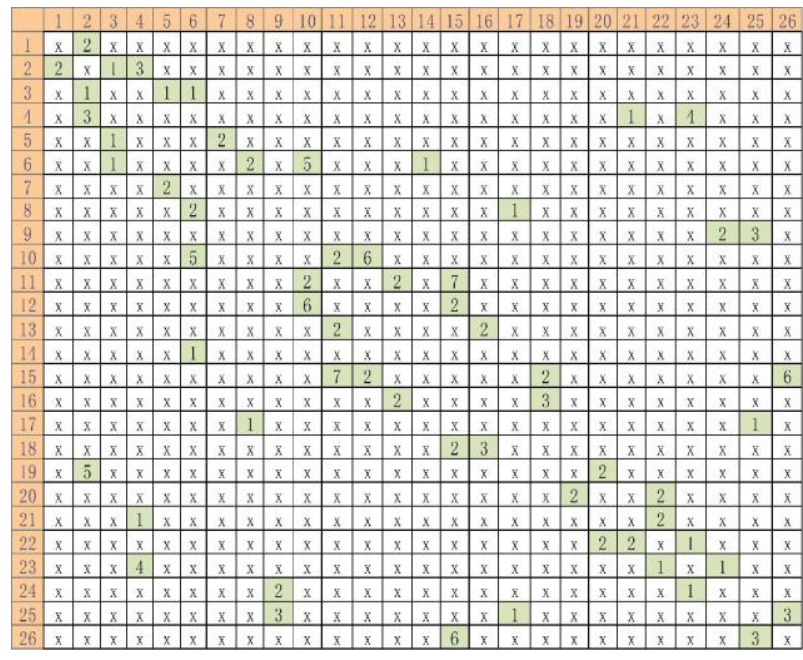

Fig. 2 Adjacency matrix

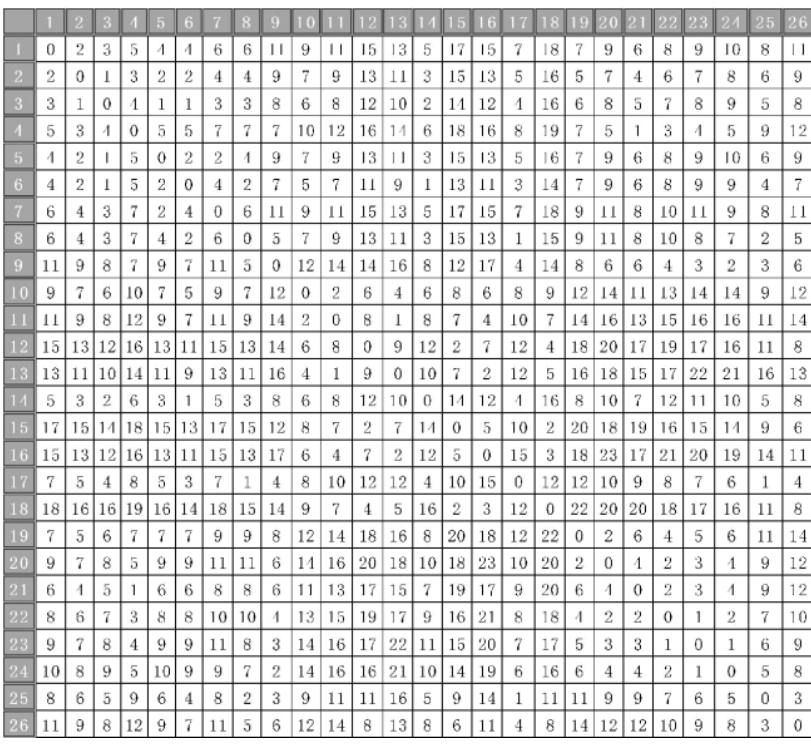

Fig. 3 Shortest path

\section{A. Symbolic description}

$N_{1}$ : The number of patrol lines with 35 minutes of inspection cycle;

$N$ : The total number of inspection lines is 35 minutes and 60 minutes.

$n_{i j}$ : During the inspection process, the number of inspection points passed through the $i$ routes ( $i=1$ is 35 minutes for the inspection cycle, and 60 minutes for the $i=2$ inspection cycle).

$W:$ The time it takes to complete a patrol.

$W_{i}$ : Time consuming for inspection of $i$ lines;

$T$ : Effective time consuming to complete one inspection.
$T_{i}$ : The effective time consuming of $i$ lines is inspecting.

$a_{i}$ : Time for inspection of $i$ checkpoints;

$t_{i j}$ : The shortest time consuming (shortest path) of $i$ inspection points to $j$ inspection points.

\section{B. The establishment of the model}

- Based on the analysis of the problem, the following mathematical models are established:

$$
\text { objective function: } \min T=\sum_{i=1}^{N} T_{i}
$$

$$
\text { Constraints: s.t. }\left\{\begin{array}{c}
0 \leq T_{i} \leq 60 \\
\sum_{i=1}^{N}\left(T_{i}-\frac{\sum_{i=1}^{N} T_{i}}{N}\right)^{2} \leq \varepsilon
\end{array}\right.
$$

Among them, $T_{i}=\sum_{i=1}^{26} a_{i} x_{i}+\sum_{j=1}^{n_{2 i}-1} \sum_{i=1}^{n_{2 i}-1} t_{b_{2 i, j}, b_{2 i, j+1}}+t_{22, i 0}$,

$$
x_{i}= \begin{cases}1, & i \in B_{2 i} \\ 0 & i \notin B_{2 i}\end{cases}
$$

$\varepsilon$ is a very small positive number, which can be adjusted properly. $B_{2 i}$ is an array of $i$ inspection lines for inspection lines, and $t_{22, i 0}$ is the shortest path between the starting point of the first patrol line and the 22 point[1][4][7].

\section{The solution of the model}

First, it is divided into 35 minute group and 60 minute group according to the inspection cycle. Starting from No. 22 repair point, First, from all inspection points, we automatically search for a 35 minute inspection cycle, and find out all routes that point 22 point 22 . Then select any point as the starting point from the remaining inspection period of 35 minutes without inspecting, and continue the automatic inspection. At the same time, the time of walking and the time of inspection, plus the shortest walking time of number 22 to the starting point are not more than 35 minutes. As the next closed loop inspection route, all points on the 35 minute inspection cycle are completed. If there is a closed loop that takes less than 35 minutes at this time, the inspection cycle of the inspection cycle of 50 is set to 35 minutes, and the patrol line is redesigned to meet the time consumption of no more than 35 minutes. Then from the inspection cycle of the inspection cycle for 60 minutes to take a point, set as the starting point, set up the inspection route of the maximum time of 60 minutes, repeat the similar operation, until all inspection points inspection end. If there is a closed loop time consuming less than 60 minutes, the inspection period of the inspection cycle with 80 minutes will be taken as 60 minutes to recalculate the inspection route. 
In the final generation of several closed loop inspection lines, the total time consumption of each group is calculated and the total time spent is the minimum route.

The algorithm is designed as follows:

- $\quad$ step 1: Initialization $T_{i}=0(0 \leq i \leq 26)$.

- $\quad$ step 2: Starting from patrol point 22, traversing all inspection points in turn, select any point associated with it as the next inspection point and calculate $T_{i}$. Point $A_{i}$ into the closed loop array $B_{2 i}$.

- $\quad$ step 3: Then continue to traverse all the remaining inspection points $A_{i}(1 \leq i \leq 21)$, select any point associated with it as the next inspection point and calculate $T_{i}$. Point $A_{i}$ into the closed loop array $B_{2 i}$.

- $\quad$ step 4: Repeat step 3 until back to the starting point (that is, patrol point 22) as a closed loop. If the total travel time $T_{i}$ is less than the inspection cycle, it will be stored in an array $B_{2 i}$ in turn. The time spent on the inspection of each point and the time spent by the general line. As long as the total time consumption is less than the inspection cycle, patrol as many as possible.

- $\quad$ step 5: Set a starting point from the remaining 35 minutes for the non inspection point, using $n_{2 i 0}$, Go through all the points in turn. select any point associated with it as the next inspection point and calculate $T_{i}$. Point $A_{i}$ into the closed loop array $B_{2 i}$.

- $\quad$ step 6: continue to traverse all the remaining points, select any point associated with it as the next inspection point and calculate $T_{i}$. Point $A_{i}$ into the closed loop array $B_{2 i}$.

- $\quad$ step 7: Turn to step 6 until you return to the starting point as the next closed loop. If $t_{22, i 0}+T_{i}<35$, Then the time spent in checking the points in the array is added to the time spent by the head office. Inspection as much as possible under $T_{i} \leq 35$ circumstances;

- $\quad$ step 8: Turn to step 5 until the inspection period is 35 minutes.

- step 9: If $T_{i} \leq 35$, the inspection period of the inspection time of 50 minutes is taken as 35 minutes to recalculate the line.

- $\quad$ step 10: From the inspection point with a residual inspection period of 60 minutes, one of the inspection points is taken as the initial point, all the inspection points are traversed in turn. select any point associated with it as the next inspection point and calculate $T_{i}$. Point $A_{i}$ into the closed loop array $B_{2 i}$.

- $\quad$ step 11: Then continue to traverse all the remaining inspection points, select any point associated with it as the next inspection point and calculate $T_{i}$. Point $A_{i}$ into the closed loop array $B_{2 i}$.

- $\quad$ step 12: Turn to step 11 until you return to the starting point as the next closed loop. If $t_{22, i 0}+T_{i}<60$, Then the time spent in checking the points in the array is added to the time spent by the head office. Inspection as much as possible under $T_{i} \leq 60$ circumstances.

- $\quad$ step 13: Turn to step 10 until all inspection points are completed.

- Take the condition $\min T=\sum_{i=1}^{N} T_{i}$ and the $N$ minimum inspection line as the final inspection line.

\section{INSPECTION SCHEME}

Inspection route for fixed work time:

Line A: 22-20-19-2-1-2-4-23-22

Line B: 22-23-24-9-25-17-8-6-3-2-4-23

Line C: 22-21-4-2-3-6-14-6-8-17-25-26-25-17-8-6

Line D: 22-21-24-2-3-6-10-11-15-12-10

Line E: 22-23-24-9-25-26-15-18-16-16-18-15

Line F: 22-21-4-2-3-6-10-11-13-16-18-15-26-25-9-24-23-22

Line G: 22-21-4-2-3-5-7-5-3-6-8-17-25-9-24-23-22

Consider the rest and meal schedule:

In order to ensure the balance of human resources, three classes in the afternoon alternate each other, eight workers in each class, eight groups of inspection routes alternate each other, that is, every 24 days for a cycle. The results of the shift are as follows:

Line A: 22-20-19-2-1-2-4-23-22

Line B: 22-23-24-9-25-17-8-6-3-2-4-23

Line C: 22-21-4-2-3-6-14-6-8-17-25-26-25-17-8-6

Line D: 22-21-24-2-3-6-10-11-15-12-10

Line E: 22-23-24-9-25-26-15-18-16-16-18-15

Line F: 22-21-4-2-3-6-10-6-3-2-4

Line G: 22-21-4-2-3-5-3-6-8-17-25-9-24-23-22

Line H: 22-21-4-2-3-5-6-10-11-10-6-8-17-25-9-24-23-22 


\section{SUMMARY}

In this paper, the methods used in the course of inspection and scheduling can be extended to the inspection cases such as the maintenance of the transmission lines and the patrol inspection cases in various walks of life, such as the electric power line inspection of unmanned aerial vehicles, and the idea and method of constructing the integer programming model, which is efficient and widely applied, not only apply to the allocation of human resources, but also be applied to the allocation of human resources. It is suitable for similar call center seat scheduling system and course scheduling system.

\section{REFERENCES}

[1] Han Zhong G. Methods and applications of mathematical modeling [C]. higher education press, 2009. (In Chinese)

[2] Chen Gang. Research on power line inspection management system [D]. Guiyang: Guizhou University, 2006:7-9. (In Chinese)

[3] Shi Yuanpeng, Jiang Jianjun, Zheng Deyu, etc, transmission line inspection path planning and modeling [J]. Applied science and technology, 2011, 38 (11):18-21. (In Chinese)

[4] Hu Yun Quan. Course of operations research (Fourth Edition). Beijing: Tsinghua University press, 2012. (In Chinese)

[5] Liu Yaguo. Graph theory of adjacency matrix [J]. Journal of Yizhou Teachers College, 2008, 24 (4): 1-5. (In Chinese)

[6] Le Yang, Gong Jian ya,.Dijkstra, a high efficient algorithm for shortest path algorithm $[\mathrm{J}]$. Journal of Wuhan University of Surveying and mapping, 1999, 24 (3):209-212. (In Chinese)

[7] Li Xuewen, Wang Hongzhou, Li Bingzhao. Selection and comment on excellent papers in mathematical modeling. (First Edition). Beijing. Tsinghua University press, 2017. (In Chinese) 